\title{
Focus on: Diagnostic and Prognosis of Severely Traumatized Patients
}

Major trauma, usually associated with hemorrhagic shock, requires the utmost effort to ensure survival and optimal rehabilitation. While the event that caused trauma cannot be influenced, and primary resuscitation depends on various circumstances, emergency room algorithms can be defined and learned. These standards, however, are influenced by advances such as those due to the introduction of new diagnostic methods (ultrasound, CT scan) $[1,2]$ or therapeutic protocols (damage control surgery) [3-6]. However, severely traumatized patients suffer from a wide variety of injury patterns, a common problem with clinical studies in this field.

Predicting survival by determining physiological parameters such as base excess, lactate and $\mathrm{pH}$ is one way of defining the severity of hemorrhagic shock. These physiological parameters, which are accounted for in trauma scores, appear to be of the greatest prognostic value when assessed during the first hour after admission, as reported in this issue by Abt et al. [7] from the Zurich Trauma Center. As well as physiological scores, anatomical scores are also very important, and combining both scores seems a reasonable approach. However, the tremendous variety of injury patterns, both anatomically and physiologically, makes them difficult to grade. A further step towards improving trauma scores is presented by Lefering [8], who describes the revised injury severity classification score (RISC), an outcome prediction score that takes the individual pattern of injury into account. This new score allows severely traumatized patients to be compared while accounting for their individual patterns. It is one of the main evaluative tools used in the German Trauma Registry, and shows great promise [1, 9-12].

Furthermore, comparisons between trauma centers can be problematic, since trauma patterns differ significantly between countries and areas [13]. In the paper of Wyen et al. [14], the injury patterns and diagnostic algorithms in an urban German university

Eur J Trauma Emerg Surg 2009;35:427-8

DOI 10.1007/s00068-009-4005-y

Published Online: September 26, 2009 trauma center are depicted. The predominance of blunt trauma and importance of diagnostic timeliness over a five-year period are discussed. In this respect, optimized diagnostic procedures are warranted to ensure that important pathologies are not overlooked. It seems that missed injuries only lead to a fatal outcome in a minority of cases, as the important study of Söderlund et al. [15] demonstrates in an eight-year evaluation of early deaths in the emergency room. In certain cases, however, missing diagnoses or interpretations could have saved individual patients.

Interestingly, optimal diagnostic procedures not only prevent early trauma deaths; they can also predict the amount of posttraumatic inflammation. Maier et al. [16] demonstrated that the initial detection of lung contusions by spiral CT caused an immediate enhanced inflammatory response in combination with severe lung dysfunction. Moreover, patients with comparable injury severities but without initial visible lung contusions developed this degree of inflammation and lung dysfunction five days later in most cases. This indicates that early CT diagnostics may also be of value in initiating appropriate intensive care treatment.

Taken together, standardized algorithms and appropriate clinical, laboratory and technical diagnostic tools must be applied during initial clinical trauma care. These data can be used in individualized prognostic trauma scores, allowing improved clinical studies in the future.

\section{Otmar Trentz, Associate Editor Ingo Marzi, Managing Editor}

\section{References}

1. Huber-Wagner S, Lefering R, Ovick LM, Korner M, Kay MV, Pfeifer KJ, Reiser M, Mutschler W, Kanz KG. Effect of wholebody CT during trauma resuscitation on survival: a retrospective, multicentre study. Lancet 2009;373:1455-61.

2. Ruesseler M, Kirschning T, Breitkreutz R, Marzi I, Walcher F. Prehospital and emergency department ultrasound in blunt abdominal trauma. Eur J Trauma Emerg Surg 2009; 35:341-346.

3. Clarke JR, Trooskin SZ, Doshi PJ, Greenwald L, Mode CJ. Time to laparotomy for intra-abdominal bleeding from trauma does affect survival for delays up to $90 \mathrm{~min}$. J Trauma 2002; $52: 420-5$. 
4. Pape HC, Grimme K, Van Griensven $M$, Sott $A H$, Giannoudis $P$, Morley J, Roise O, Ellingsen E, Hildebrand F, Wiese B, Krettek C. Impact of intramedullary instrumentation versus damage control for femoral fractures on immunoinflammatory parameters: prospective randomized analysis by the EPOFF Study Group. J Trauma 2003;55:7-13.

5. Taeger G, Ruchholtz S, Waydhas C, Lewan U, Schmidt B, Nast-Kolb D. Damage control orthopedics in patients with multiple injuries is effective, time saving, and safe. J Trauma 2005;59:409-16; discussion 417.

6. Pape HC, Rixen D, Morley J, Husebye EE, Mueller M, Dumont C, Gruner A, Oestern HJ, Bayeff-Filoff M, Garving C, et al. Impact of the method of initial stabilization for femoral shaft fractures in patients with multiple injuries at risk for complications (borderline patients). Ann Surg 2007;246:491-9; discussion 499-501.

7. Abt R, Lustenberger T, Stover JF, Benninger E, Lenzlinger PM, Stocker R, Keel M. Base excess determined within one hour of admission predicts mortality in patients with severe pelvic fractures and severe hemorrhagic shock. Eur J Trauma Emerg Surg 2009;35:429-36.

8. Lefering R. Development and validation of the revised injury severity classification score for severely injured patients. Eur J Trauma Emerg Surg 2009;35:437-47.

9. Wutzler S, Maegele M, Marzi I, Spanholtz T, Wafaisade A, Lefering R. Association of preexisting medical conditions with in-hospital mortality in multiple-trauma patients. J Am Coll Surg 2009;209:75-81.
10. Ruchholtz S, Lefering R, Paffrath T, Oestern HJ, Neugebauer E, Nast-Kolb D, Pape HC, Bouillon B. Reduction in mortality of severely injured patients in Germany. Dtsch Arztebl 2008;105:225-231.

11. Lefering R. Trauma score systems for quality assessment. Eur J Trauma 2002;28:52-63.

12. Deutsche Gesellschaft für Unfallchirurgie, Sektion Intensiv- \& Notfallmedizin, Schwerverletztenversorgung (NIS). Jahresbericht 2008 für den Zeitraum bis Ende 2007 für DGU Traumaregister der Sektion NIS - DGU gesamt. http://www. traumaregister.de, 2008.

13. MacKenzie EJ, Rivara FP, Jurkovich GJ, Nathens AB, Frey KP, Egleston BL, Salkever DS, Scharfstein DO. A national evaluation of the effect of trauma-center care on mortality. N Engl J Med 2006;354:366-78.

14. Wyen $\mathrm{H}$, Wutzler $\mathrm{S}$, Rüsseler $M$, Mack $M$, Walcher F, Marzi I. Five years experience of trauma care in a German Urban Level I University Trauma Center. Eur J Trauma Emerg Surg 2009;35:448-54.

15. Söderlund T, Tulikoura I, Niemelä M, Handolin L. Traumatic deaths in the emergency room: a retrospective analysis of 115 consecutive cases. Eur J Trauma Emerg Surg 2009;35:455-62.

16. Maier M, Geiger EV, Wutzler S, Lehnert $M$, Wiercinski A, Buurman WA, Marzi I. Role of lung contusions on posttraumatic inflammatory response and organ dysfunction in traumatized patients. Eur J Trauma Emerg Surg 2009;35:463-9. 